\title{
Peak Shaving through closed-loop optimization applied to machine tools with periodic behaviour
}

\author{
Sergi Xavier Ubach Pallas ${ }^{1}$, Jenny L. Diaz C. ${ }^{1}$, Carlos Ocampo-Martinez ${ }^{1}$, Senior Member, IEEE, Miguel Antunez ${ }^{2}$ \\ ${ }^{1}$ Universitat Politècnica de Catalunya, Institut de Robotica i Informàtica Industrial (CSIC-UPC), \\ Llorens i Artigas 4-6, Planta 2, Barcelona 08028, Spain \\ ${ }^{2}$ Plethora IIoT, San Antolin 3, 20870 Elgoibar, Spain \\ Corresponding author: subach@iri.upc.edu
}

\begin{abstract}
This paper deals with machine tools characterized by a periodic behaviour and high-power peaks that require an oversized electrical network and cause high costs because electrical utilities charge greatly each power peak. To solve this issue, a new peak-shaving methodology is proposed based on polynomial models and optimization, to reduce the power-consumption peaks height in machine tools with periodic behaviour. A test-bench that emulates the electrical behaviour of a machine tool is used in order to test the proposed method with real data. In the scenarios simulated, the peak height has been reduced between $35 \%$ and $15 \%$.
\end{abstract}

\section{INTRODUCTION}

Manufacturing industry is one of the most important pieces of the worldwide economy. Employing hundreds of millions of workers, it has great impact on the society and the environment. That is because during the last years there has been an increasing interest in the efficient energy management [1]-[3].

The main reason for considering a suitable energy management is related to the ecological impact, a growing point of concern and a clear tendency in the future. Governments are generating new taxes for electrical consumption, which is not strange given that more than $60 \%$ of the electricity of the world is consumed by industry [4]. However, there is also another reason for companies to pursue energy efficient methods: reducing production cost.

This interest has led groups from all the world to invest time and resources towards proposing more efficient ways to reach consumption reduction [5]. As a first categorization on how the efficiency problem in machine tools is approached, available techniques are divided into two clearly differentiated subcategories:

1) Machine-tool design: A thoughtful analysis on the mechanical design of the machine will reduce considerably the amount of power needed. Some of the proposed options would be redistributing weights, lightening the mobile parts or modifying the shape or material of the end effector [6]. The materials used in the produced part can also reduce the power required for the same task. Finally, in [7], a Kinetic Energy Recovery System (KERS) is proposed, allowing an overall reduction of the

2017 IEEE 3rd Colombian Conference on Automatic Control (CCAC) consumption by generating energy from the machining process.

2) Process planning: This category is focused on how the process is organized. Some authors propose solutions relative to switching the work state of the machine when it is idle [8], [9], while other authors use either logical or mathematical methods to optimize the order and execution time of the elements such that the overall consumption of the machine tool is minimized.

Both methods mentioned above have plenty of possibilities, but in this document, only the process planning is going to be addressed as, in most cases, modifying a machine is not a possibility. According to [10]-[13] and [14], in order to reduce consumption, the secondary systems can be turned off at some time instants and some methods to detect those instants are presented (e.g., cooling, air pumps).

In [3], [6], [7], [13], it is demonstrated that accelerating the spindle or cutting in less time will contribute to reducing the resources spent at the expense of increasing the load in critical instants of time.

However reducing the power consumption will not necessarily reduce the production cost. The price of electricity depends on the usage and on the maximum power contracted [15]. Using techniques that reduce consumption in exchange of generating big peaks can turn into extra expenses if these peaks are not managed properly.

Peak shaving is the process of reducing the amount of energy purchased from the utility company during peakdemand hours. Usually focused on reducing home electrical bill by redistributing the big power spenders during the day and turning off unnecessary secondary systems [16], peak shaving can be applied to machine tools with periodic behaviour in order to fit the most power-demanding elements (e.g., drills, motors) and turning off secondary systems when they are not required. A common solution is to add capacitors to the system in order to soften the peak [17].

The main contribution of this paper is a solution that reduces the power consumption peaks by analyzing the behaviour of periodic machine tools and rearranging the activation sequence so that the systems minimizes the maximum instantaneous consumption. In contraposition to methods for peak shaving that use approaches in the field of machine-tool design or state- 
machine solutions, the methodology proposed in this paper optimizes the order and execution time of the elements in order to minimize the height of the maximum peak in power consumption.

In order to comply the aim of this work, the problem is going to be defined in a more precise way and the restrictions that influence the operation of the machine tools under study in Section II. Afterwards, the proposed approach is going to be explained in Section III, including the assumptions made, the optimization method and an explanation about the models used. In Section IV, the results obtained are going to be presented through two test scenarios and a study on which model identification algorithm had better results with the given criteria. Finally, in Section V the conclusions of this paper are presented together with the lines of future research.

\section{PROBlEM FORMULATION}

This paper considers periodic behaviour machine tools with a fixed period length $T_{p}$ and fixed execution length $T_{e_{n}}$ for each element, which are controlled by binary (on/off) activation signals.

An activation signal is defined by $\delta_{e_{n_{-}} o n}$, which represents the activation time of the $n$ element in the machine tool. The activation sequence will be then defined as a set of activation and deactivation signals $\left(\delta_{e_{n_{-}} \text {off }}\right)$ $\Lambda=\left\{\delta_{e_{1} \_n}, \delta_{e_{1 \_} \text {off }}, \delta_{e_{2_{-} o n}}, \delta_{e_{2 \_} o f f}, \ldots, \delta_{e_{n_{-} o n}}, \delta_{e_{n_{-}} o f f}\right\}$. Given the constraint that the execution time of each element is constant, then $\delta_{e_{n_{-}} \text {off } f}=\delta_{e_{n_{-}} \text {on }}+T_{e_{n}}$, which means that the sequence can be simplified for this problem as pairs of activation time and length of the element, i.e.,

$$
\Lambda=\left\{\left(\delta_{e_{1}}, T_{e_{1}}\right),\left(\delta_{e_{2}}, T_{e_{2}}\right), \ldots,\left(\delta_{e_{n}}, T_{e_{n}}\right)\right\},
$$

with $\delta_{e_{n}}=\delta_{e_{n_{-}} \text {on }} \in\left(0, T_{p}-T_{e_{n}}\right)$ and $T_{e_{n}}<T_{p}$. In Figure 1, a diagram of machine tool with an activation signal $(\Lambda)$ and an apparent power output $(S)$ is proposed.

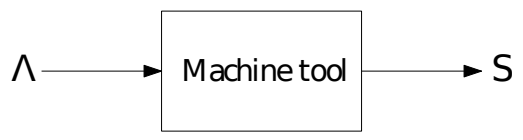

Figure 1: Open loop general scheme with inputs and outputs

Therefore, the objective of peak-shaving techniques consists in restricting the machine tool's apparent power consumption under a certain value, i.e.,

$$
T_{h}>S(k, \Lambda),
$$

being $T_{h}$ a threshold determined by the maximum value the power consumption peaks may reach, $k \in \mathbb{Z}_{\geq 0}$ the discrete time and $S(k, \Lambda)$ the machine tool's apparent power consumption in all three phases, in function of $k$ and $\Lambda$. It should be noted that the apparent power $(S)$ can be defined as the modulus of active $(P)$ and reactive $(Q)$ power in VoltAmperes, and given by $S=P+j Q$ [18].

\section{PROPOSED APPROACH}

Based on the problem formulation, the only variables left are $\delta_{e_{n}}$ that, from now on, are going to be treated as the inputs of the system.

The proposed solution consists in optimizing $\delta_{e_{n}}$ values in (1) to minimize the peaks, leading to a solution for (2). Since $S$ in (2) refers to the apparent power consumption of the real machine tool, it must be modeled to use the output as the function to minimize for the optimization problem.

For this preliminary approach, the assumptions below should be considered.

Assumption 1: The order in which $\delta_{e_{n}}$ are activated can be modified.

Assumption 2: Activating two elements at the same time instant is possible.

Assumption 3: All the elements have the same priority when activation signal is sent.

Assumption 4: Each element activates only once each period $T_{p}$.

\section{A. Optimization}

Machine tools are operated by a defined sequence of its elements, each one with an activation and deactivation time, and the total operation time of the sequence known as period $\left(T_{p}\right)$. Therefore, and taking into account the periodic behavior of this process, it can be considered as a batch process with each period equal to a batch, in which for an activation sequence of their elements, the machine operation is defined during a period [19].

In this way, the resulting optimization problem is stated as follows:

$$
\begin{array}{cl}
\min _{\Lambda} & J=\left\|M\left(\Lambda^{\prime}\right)\right\|_{\infty} \\
\text { s.t. } & \Lambda \in\{0,1\} \times \mathbb{R}_{+}, \\
& \forall k \in\left[0, T_{p}\right],
\end{array}
$$

being $M$ the model of the machine tool defined in Section III-B and $\Lambda^{\prime}$ a set of discrete signals, one for each element in the machine tool and $J$ corresponds with the cost function to be minimized. Each signal in $\Lambda^{\prime}$ is a binary sequence that has the same sampling time that the model and a period duration of $T_{p}$. It will have a null logical value for the whole duration, except from $\delta_{e_{n}}$ [s] until $T_{e_{n}}$ [s], in which the signal has a value of logical one. In Figure 3, an example of two of signals forming a $\Lambda^{\prime}$ is shown.

The optimization algorithm proposed is pattern search [20]. It varies a single theoretical parameter, in this case $\delta_{e_{n}}$, at a time by steps of the same magnitude. When no such increase or decrease in any $\delta_{e_{n}}$ reduces (3), it halves the step size and repeats the process until the steps are deemed sufficiently small. Once a minimum is found, the optimal sequence found is defined as $\Lambda^{*}$.

Due to the delay between activation signal and actual activation of the actuator, there will be cases when an element is not turned off by the end of the period $T_{p}$. This would cause that the initial conditions for the subsequent periods would 
be different than the original, causing unexpected results, such as higher peaks. To avoid this behaviour, two periods are considered when solving the optimization problem in (3) instead of just one. This way transitions between periods will be taken into consideration in the optimization and thus avoid undesired overlapping.

The initial condition of the optimization problem $\left(\Lambda_{0}^{\prime}\right)$ is the sequence $\Lambda^{\prime}$ that made the machine-tool apparent power $S\left(\Lambda^{\prime}\right)$ go over the threshold. If the optimal sequence $\Lambda^{*}=\Lambda_{0}$, the solution of (3) corresponds with a local minimum of the cost function $J$ (also in (3)) and it would keep violating the threshold with the new sequence. In order to find a better solution, the optimization problem is launched again, this time with a randomly generated initial condition $\Lambda_{0}$ such that its components are defined as

$$
\delta_{e_{n}}=\operatorname{rand}\left(0, T_{p}-T_{e_{n}}\right),
$$

if a solution is not found either, $\Lambda_{0}$ will update with new randomly generated sequences until

$$
S\left(\Lambda^{*}\right)<S\left(\Lambda_{0}^{\prime}\right)
$$

or a time out proportional to the $T_{p}$ triggers. In case of not finding a better solution, the machine-tool will continue on using $\Lambda^{*}=\Lambda_{0}^{\prime}$.

In Figure 2, a diagram of the closed-loop optimization-based approach is shown, where the optimization module solves (3) with the received initial conditions and constraints. The optimization solution sequence $\Lambda^{*}$, is then validated on the plant and fed back to the optimization algorithm to analyze its suitability and to propose a new one if suited.

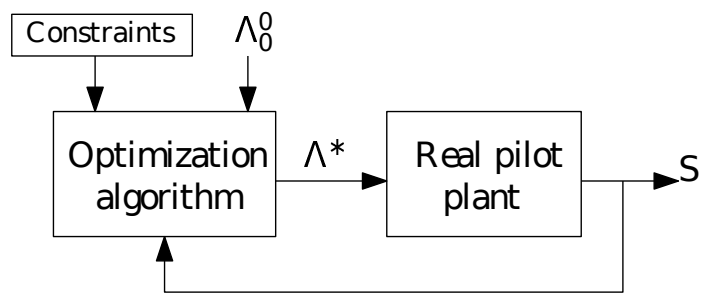

Figure 2: Diagram of the closed-loop topology

\section{B. Model identification}

Apparent power consumption of the model will be used as the cost function $J$ in (3). The main criterion to decide which model is the most suitable will be the fitting or how similar the model output is to the real machine tool response with the same inputs. Training data for the model consists in a $\Lambda$ with all the possible combinations of sequence of the $n$ elements, taking into account Assumptions 1 to 4. In Figure 3, a simple example for two inputs is shown.

Generally, a discrete system can be described by using the general-linear polynomial model [21] as follows:

$$
A(z) y(k)=\frac{B(z)}{F(z)} u(k)+\frac{C(z)}{D(z)} w(k),
$$
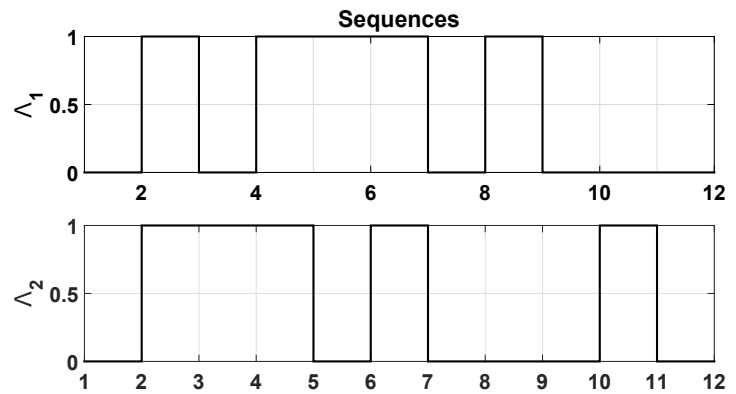

Figure 3: Example of modeling sequence

where $y(k)$ are the system outputs, $u(k)$ are the system inputs and $w(k)$ is the system disturbance. $A(z), B(z), C(z), D(z)$, and $F(z)$ are polynomial in function of the backward shift operator $z-1$ and defined by the following expressions:

$$
\begin{aligned}
& A(z)=1+a_{1} z^{-1}+\cdots+a_{k_{a}} z^{-k_{a}}, \\
& B(z)=b_{0}+b_{1} z^{-1}+\cdots+b_{k_{b}} z^{-k_{b}}, \\
& C(z)=1+c_{1} z^{-1}+\cdots+c_{k_{c}} z^{-k_{c}}, \\
& D(z)=1+d_{1} z^{-1}+\cdots+d_{k_{d}} z^{-k_{d}}, \\
& F(z)=1+f_{1} z^{-1}+\cdots+f_{k_{f}} z^{-k_{f}},
\end{aligned}
$$

with $a_{i}, b_{i}, c_{i}, d_{i}$ and $f_{i}$ being parameters obtained through the modeling methods explained below, and $\left\{k_{a}, k_{b}, k_{c}, k_{e}, k_{f}\right\} \in$ $\mathbb{N}_{\geq 1}$ are the order of the system. If there are multiple inputs, there can be multiple instances of $B$ and $F$.

Some specific cases of (6) are going to be analyzed and, in order to get the best possible fitting, different orders are tested.

1) $A R X$ : When $C(z), D(z)$, and $F(z)$ equal 1, (6) turns into an autoregressive with exogenous terms model (ARX), which is the simplest model that incorporates the stimulus signal [21]. However, the ARX model captures some of the stochastic dynamics as part of the system dynamics. It can be defined as

$$
A(z) y(k)=B(z) u(k)+w(k) .
$$

2) ARMAX: When $D(z)$ and $F(z)$ equal $1,(6)$ turns into an autoregressive-moving average with exogenous terms model (ARMAX). Unlike the ARX model, the system structure of an ARMAX model includes the stochastic dynamics [21]. ARMAX models are useful when dominating disturbances appear early in the process, such as at the input. The ARMAX mathematical expression is

$$
A(z) y(k)=B(z) u(k)+C(z) w(k) .
$$


3) $O E$ : When $A(z), C(z)$, and $D(z)$ equal 1, (6) turns into an output-error model (OE), which describes the system dynamics separately from the stochastic dynamics [21]. The output-error model does not use any parameter for simulating the disturbance characteristics, i.e.,

$$
y(k)=\frac{B(z)}{F(z)} u(k)+w(k) .
$$

4) $B J$ : When $A(z)$ equals 1 , (6) turns into a Box-Jenkins model (BJ) that provides a complete model of a system since it represents disturbance properties separately from system dynamics [21]. This model is useful when disturbances appear late in the process, such as measurement noise on the output, i.e.,

$$
y(k)=\frac{B(z)}{F(z)} u(k)+\frac{C(z)}{D(z)} w(k) .
$$

\section{Simulations}

\section{A. Case study description}

The proposed method has been tested in a real custom test-bench built to simulate a machine tool. As it can be seen in Figure 4, it is composed by three loads powered with a triphasic supply, a heater acting as a resistive load, a motor acting as inductive load and two uninterruptible power supplies considered as a single capacitive load. An integrated PC controls a relay (grey box in Figure 4) that will activate and deactivate the loads depending on the sequence sent by the PC. Through an acquisition tool (also in the grey box in Figure 4), the PC receives each $10 \mathrm{~ms}$ the value of $S$ [VA] from each phase. For this case, the data under study will be the sum of these three values of $S$.

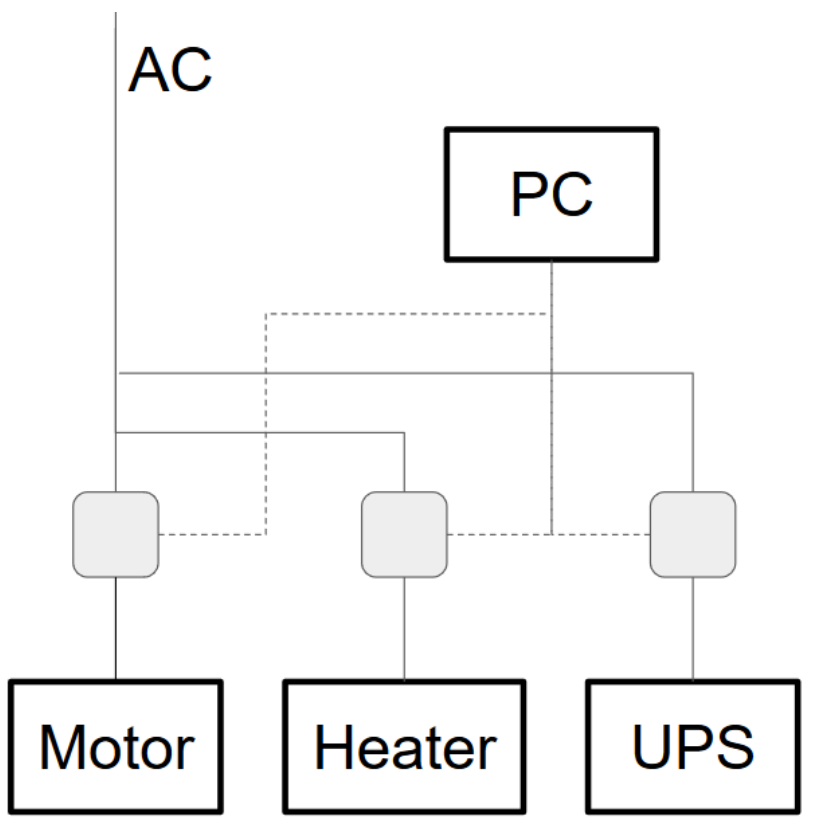

Figure 4: Test-bench used to simulate a machine tool

In this work, two test scenarios are studied. The sequences considered are given by

$$
\begin{gathered}
\Lambda_{1}=\{(1 \mathrm{~s}, 5 \mathrm{~s}),(1 \mathrm{~s}, 5 \mathrm{~s}),(1 \mathrm{~s}, 5 \mathrm{~s})\}, \\
\Lambda_{2}=\{(0 \mathrm{~s}, 5 \mathrm{~s}),(0 \mathrm{~s}, 15 \mathrm{~s}),(10 \mathrm{~s}, 10 \mathrm{~s})\},
\end{gathered}
$$

with the periods of each case equal to $T_{1}=26 \mathrm{~s}$ and $T_{2}=21 \mathrm{~s}$, while the thresholds $T_{h 1}=2000 \mathrm{VA}$ and $T_{h 2}=2500 \mathrm{VA}$.

\section{B. Model selection}

Defined the machine tool apparent power $S$ as the output to minimize through optimization (see problem in (3)), a model of this apparent power is required as optimizing over the real machine tool would take a lot of time. To do that, the sequence $\Lambda^{\prime}$ appearing in Figure 5 is defined.

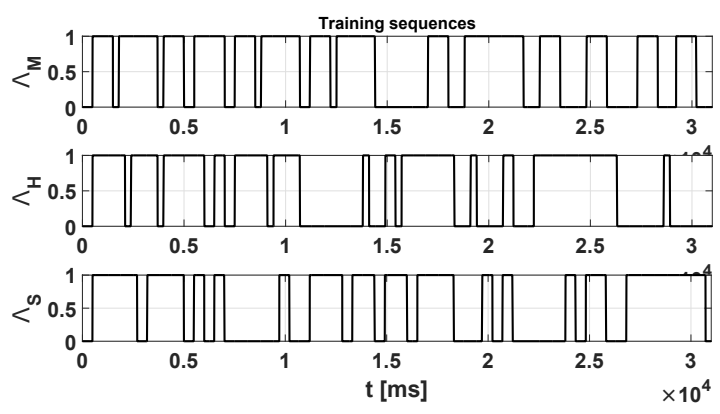

Figure 5: Input sequence

Using Matlab System Identification Tool [22], the four models mentioned in Section III are analyzed for orders from 4 to 8 and displayed in Table I, which lists the fitting for each combination of model/order.

Table I: Model fitting comparative in percentage

\begin{tabular}{c|c|c|c|c|c} 
Order & 4 & 5 & 6 & 7 & 8 \\
\hline ARX & 83.69 & 83.69 & 82.92 & 83.80 & 83.98 \\
ARMAX & 81.50 & 83.35 & 76.98 & 82.39 & 77.07 \\
\hline OE & 3.390 & -235.4 & -116.4 & 77.21 & 88.64 \\
BJ & 3.440 & -232.2 & -107.1 & 77.23 & 88.64
\end{tabular}

For each case, the model with the best fitting is selected among ARX and ARMAX of order 7 and OE and BJ of order 8 , and compared against the validation data in Figure 6. A region is zoomed to help seeing the differences.

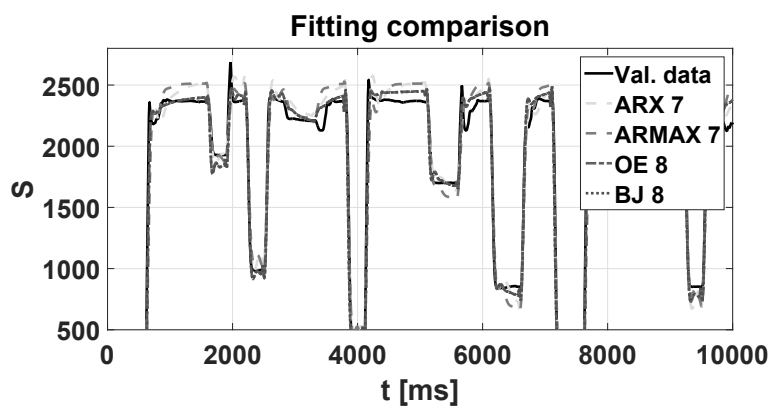

Figure 6: Models fitting 
Table II: Sequences $\Lambda_{1}^{*}$ after optimization

\begin{tabular}{c|l|l|l|l} 
& $\delta_{M}[\mathrm{~ms}]$ & $\delta_{H}[\mathrm{~ms}]$ & $\delta_{S}[\mathrm{~ms}]$ & $\max S\left(\Lambda_{1}^{\prime}\right)$ \\
\hline ARX Order 7 & 16392 & 11256 & 17384 & 1618 VA \\
ARMAX Order 7 & 17384 & 11752 & 17384 & $1597.1 \mathrm{VA}$ \\
\hline OE Order 8 & 5548 & 12392 & 17384 & 1617.4 VA \\
BJ Order 8 & 7688 & 12264 & 17464 & $1605.1 \mathrm{VA}$
\end{tabular}

\section{Test scenario 1}

In the first test scenario, the machine tool will be fed with the sequence (7a), from which the output in Figure 7 is obtained, with $\max S\left(\Lambda_{1}^{\prime}\right)=2798.4 \mathrm{VA}$.

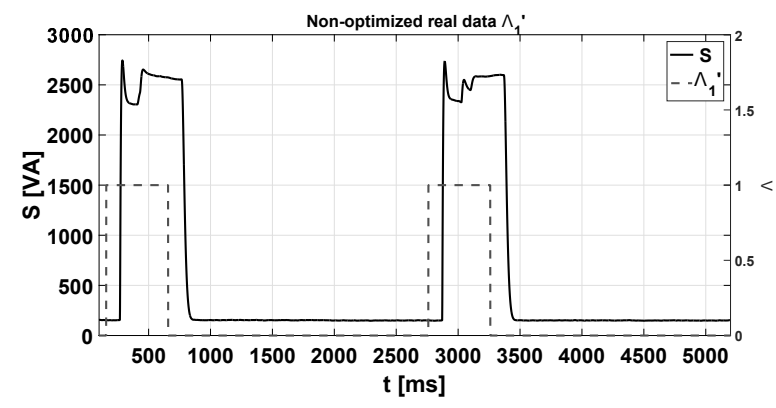

Figure 7: Machine tool output to $\Lambda_{1}$ in (7a)

The optimization has been implemented in Matlab using the pattern search algorithm function patternsearch (). In minimizing the function defined by the models with the sequence in (7a) as input, a set of new $\delta_{e_{n}}$ is obtained for each model, which will minimize the instantaneous power consumed. These results are presented in Table II together with the new maximum of $S$.

Two clearly differentiated shapes can be obtained from these results. ARX and ARMAX define similar optimized sequences, e.g., $\Lambda_{1, A R X}^{*}$ being a sequence where the two less spending activation signals (motor and UPS) are launched at the same time. On the other hand, OE and BJ define $\Lambda_{1, B J}^{*}$, a sequence where each element is active when the other two are turned off.

Figure 8 shows the approach applying an ARX model of order seven before minimizing the apparent power, while in Figure 9 the response after the optimization, is shown. When $\Lambda_{1, A R X}^{*}$ is used as input sequence in the real machine tool, the consumption obtained is the one displayed in Figure 10, with $\max S\left(\Lambda_{1, A R X}^{*}\right)=1728.6 \mathrm{VA}$.

Even though max $S\left(\Lambda_{1, A R X}^{*}\right)$ from the real plant is bigger than the $\max S\left(\Lambda_{1, A R X}^{*}\right)$ obtained from the ARX model, showing that the model has room to be improved, the results in terms of peak shaving yield a reduction of $38.23 \%$ in the height of peaks in $S$.

If the proposed approach considers the BJ model of order eight, the result before optimizing will be the one appearing in Figure 11 and the optimized response is the one appearing in Figure 12. The resulting $\max S\left(\Lambda_{1, B J}^{*}\right)$ will be of similar amount with respect to the ARX one. When this $\Lambda_{1, B J}^{*}$ solution is sent to the real machine tool, it gives the output showed in Figure 13, with $\max S\left(\Lambda_{1, B J}^{*}\right)=2191.9$ VA.

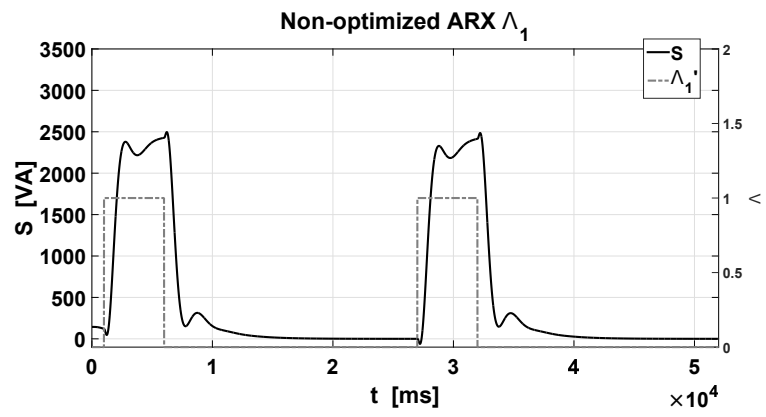

Figure 8: Simulation result for ARX order 7 facing $\Lambda_{1, A R X}^{\prime}$

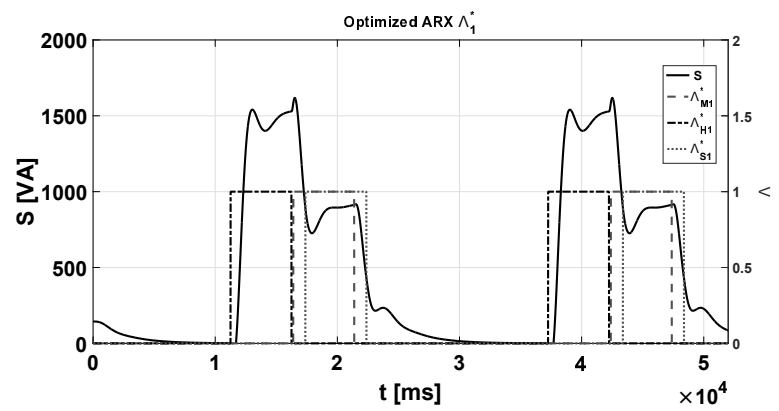

Figure 9: Simulation result for ARX order 7 facing $\Lambda_{1, A R X}^{*}$

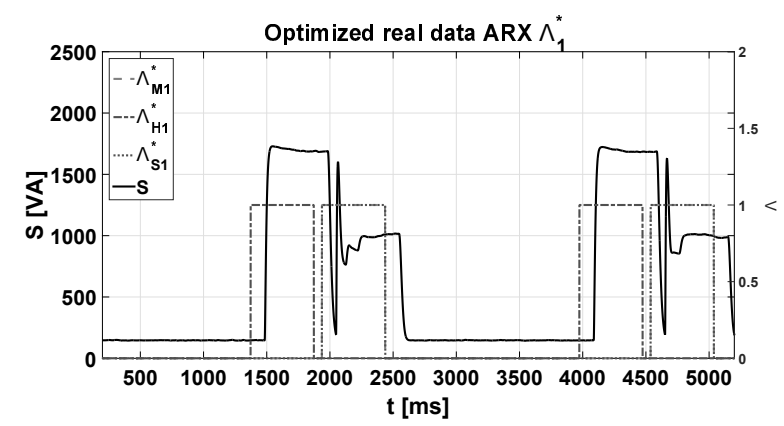

Figure 10: Machine tool output to $\Lambda_{1, A R X}^{*}$

BJ model gives worse result than the ARX as the maximum values of $S$ in simulation and real plant are more different. Although, the optimization-based approach still finds a solution that reduces the height of peaks in $S$ by $21.67 \%$.

These results are possible because this test scenario is prepared to be friendly with the proposed method and has a lot of room to reduce $\max S\left(\Lambda_{1}\right)$. In the next test scenario, the situation will be the opposite.

\section{Test scenario 2}

The test scenario 2 defines a more saturated environment without much room to maneuver with the activation sequence defined in (7b). In Figure 14, the response of the machine tool test-bench to $\Lambda_{2}^{\prime}$ is presented, with $\max S\left(\Lambda_{2}^{\prime}\right)=2699$ VA.

In Table III the values of $\Lambda_{2}^{*}$ when optimizing this case using the ARX and BJ models are shown. BJ finds a minimum of $\max S\left(\Lambda_{2 B J}^{\prime}\right)=2294 \mathrm{VA}$ close to the original $\max S\left(\Lambda_{2}^{\prime}\right)=2699 \mathrm{VA}$, this value is a local minimum and 


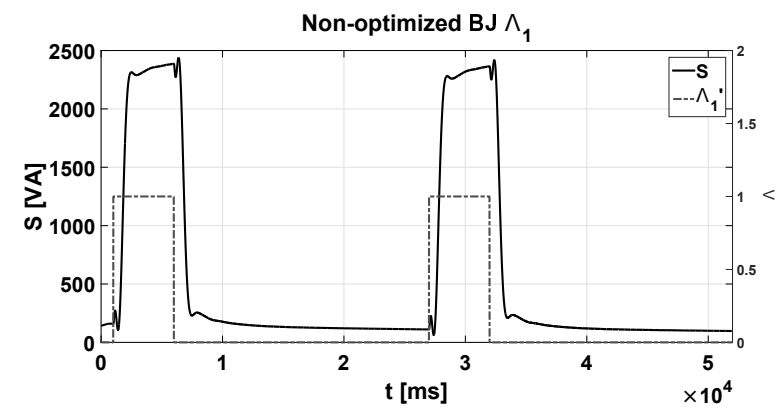

Figure 11: Simulation result for BJ order 8 facing $\Lambda_{1, A R X}^{\prime}$

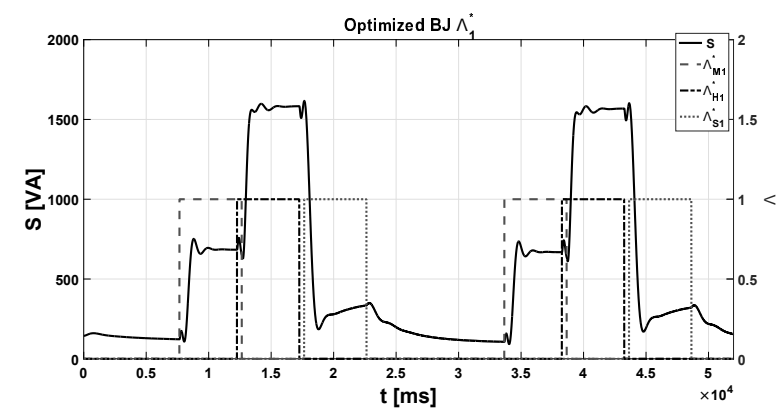

Figure 12: Simulation result for BJ order 8 facing $\Lambda_{1, B J}^{*}$

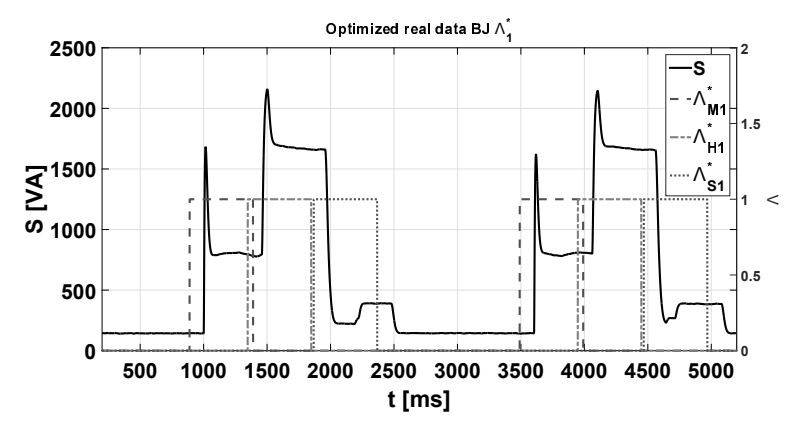

Figure 13: Machine tool output to $\Lambda_{1, B J}^{*}$

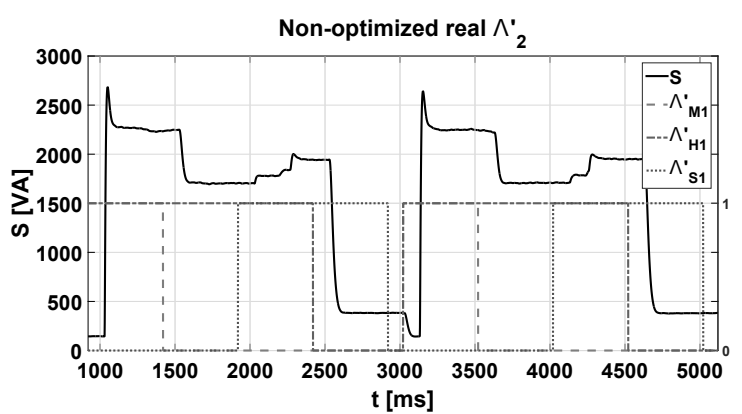

Figure 14: Machine tool output to $\Lambda_{2}$

solves the optimization problem (3). In Figure 15, the result of the ARX model solution for $S\left(\Lambda_{2, A R X}^{*}\right)$ are shown with a clear improvement in regards to Figure 14, unlike in Figure 16 , that the results for $S\left(\Lambda_{2, B J}^{*}\right)$ are very similar to the results before the optimization.

When $\Lambda_{2, A R X}^{*}$ and $\Lambda_{2, B J}^{*}$ are used on the machine
Table III: Sequences $\Lambda_{2}^{*}$ after optimization

\begin{tabular}{c|l|l|l|l} 
& $\delta_{m}[\mathrm{~ms}]$ & $\delta_{h}[\mathrm{~ms}]$ & $\delta_{s}[\mathrm{~ms}]$ & $\max S\left(\Lambda_{2}^{\prime}\right)$ \\
\hline ARX Order 7 & 1043 & 5996 & 11 & 1846 VA \\
BJ Order 8 & 97 & 204 & 1100 & 2276 VA
\end{tabular}

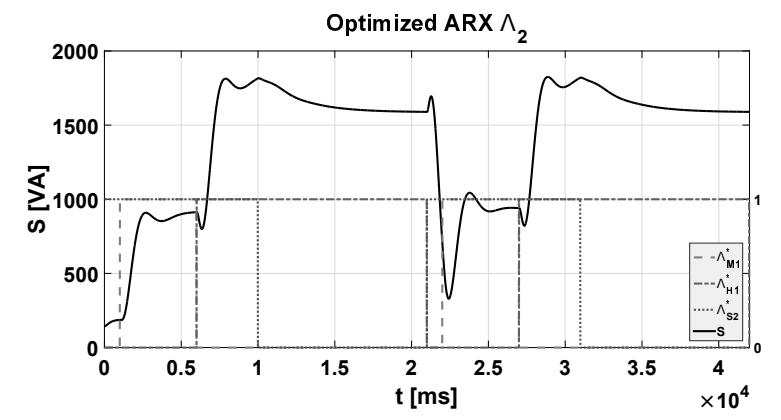

Figure 15: Simulation result for ARX ord. 7 to $\Lambda_{2, A R X}^{*}$

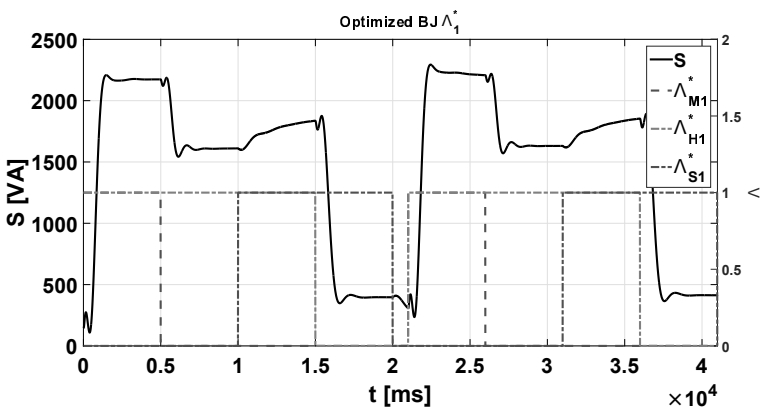

Figure 16: Simulation result for BJ ord. 8 to $\Lambda_{2, B J}^{*}$

tool-test bench, the results in Figures 17 and 18 are obtained respectively, with $\max S\left(\Lambda_{2, A R X}^{*}\right)=2010.9 \mathrm{VA}$ and $\max S\left(\Lambda_{2, B J}^{*}\right)=2276.2 \mathrm{VA}$. In this scenario, the method reduces the peak height in $25.49 \%$ with the ARX and $15.67 \%$ with the BJ. As in the Scenario 1, the ARX model method has a better performance reducing the peak both in reality and in the modeled version, but the BJ model has obtained a better resemblance between the real output an the modeled one than the ARX model.

After these two test scenarios, it can be said that the ARX model has the most suitable performance for the plant under test.

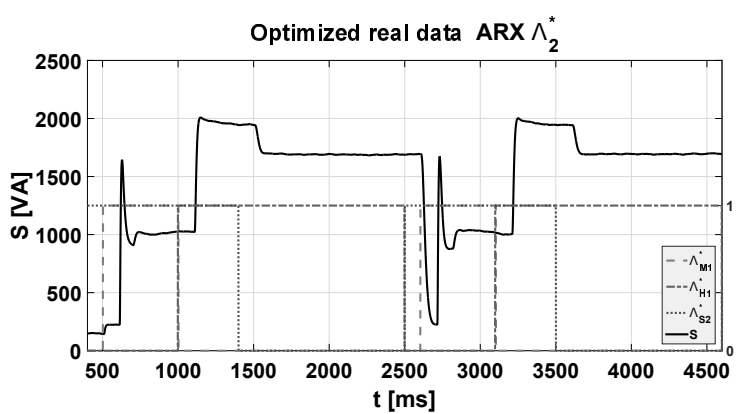

Figure 17: Machine tool output to $\Lambda_{2-A R X}^{*}$ 


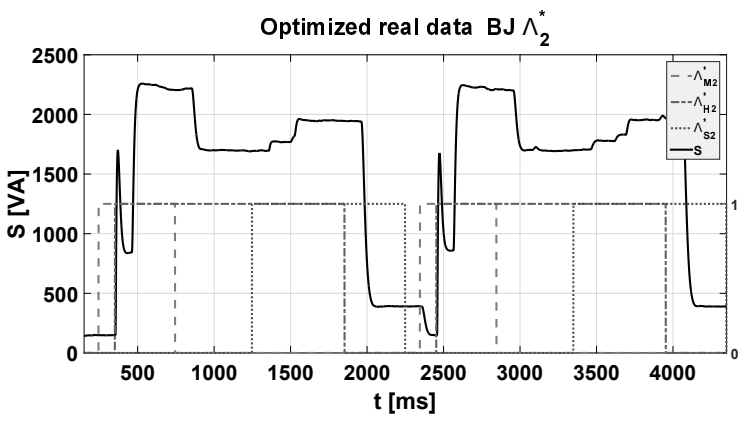

Figure 18: Machine tool output to $\Lambda_{2-B J}^{*}$

\section{CONCLUSION}

This paper has proposed a new peak-shaving approach based on polynomial models and optimization-based control to reduce the power consumption peaks height in machine tools with periodic behaviour. Both effectiveness and efficiency of the proposed approach have been discussed using a test-bench that emulates the behaviour of the kind of machine tools under study, and concluded that for these concrete case the ARX model method is the best suited. The resulting reductions range from $15.67 \%$ to $38.23 \%$ in the most favorable case, but it is important to remark that these values are subject to the amount of elements in the machine tool and the power consumption from them.

Future work extending the topics discussed in this paper will be focused on refuting Assumptions 1, 3 and 4 in order to make the method compatible with a wider range of more complex real machine tools.

\section{ACKNOWLEDGMENT}

Authors would like to thank Ikergune (Exte-Tar Group) and the project IKERCON ref. c-10683. for their scientific support in this work.

\section{REFERENCES}

[1] S. Dhanik, P. Xirouchakis, and R. Perez, A System for Resource Efficient Process Planning for Wire EDM. Berlin, Heidelberg: Springer Berlin Heidelberg, 2011, pp. 219-224.

[2] E. Wahl, K. Vincke, and M. Himmelsbach, "Recovery of energy from a laser machining system," Feb. 2011.

[3] M. Mori, M. Fujishima, Y. Inamasu, and Y. Oda, "A study on energy efficiency improvement for machine tools," CIRP Annals - Manufacturing Technology, vol. 60, no. 1, pp. 145 - 148, 2011.

[4] U. E. I. Administration, "The international energy outlook 2016," Tech. Rep., 2016.

[5] J. R. Duflou, J. W. Sutherland, D. Dornfeld, C. Herrmann, J. Jeswiet, S. Kara, M. Hauschild, and K. Kellens, "Towards energy and resource efficient manufacturing: A processes and systems approach," CIRP Annals - Manufacturing Technology, vol. 61, no. 2, pp. 587 - 609, 2012.

[6] G. Campatelli, L. Lorenzini, and A. Scippa, "Optimization of process parameters using a response surface method for minimizing power consumption in the milling of carbon steel," Journal of Cleaner Production, vol. 66, pp. $309-316,2014$.

[7] N. Diaz, M. Helu, A. Jarvis, S. Tönissen, D. Dornfeld, and R. Schlosser, "Strategies for minimum energy operation for precision machining," Laboratory for Manufacturing and Sustainability, 2009.

[8] N. Weinert, S. Chiotellis, and G. Seliger, "Methodology for planning and operating energy-efficient production systems," CIRP Annals Manufacturing Technology, vol. 60, no. 1, pp. 41 - 44, 2011.
[9] S. Newman, A. Nassehi, R. Imani-Asrai, and V. Dhokia, "Energy efficient process planning for cnc machining," CIRP Journal of Manufacturing Science and Technology, vol. 5, no. 2, pp. 127 - 136, 2012.

[10] P. Eberspchera and A. Verla, "Realizing energy reduction of machine tools through a control-integrated consumption graph-based optimization method," Procedia CIRP, vol. 7, pp. 640 - 645, 2013.

[11] E. Abele, N. Panten, and B. Menz, "Data collection for energy monitoring purposes and energy control of production machines," Procedia CIRP, vol. 29, pp. $299-304,2015$.

[12] N. Frigerio and A. Matta, "Energy efficient control strategy for machine tools with stochastic arrivals and time dependent warm-up," Procedia CIRP, vol. 15 , pp. $56-61,2014$.

[13] J. Hesselbach and C. Herrmann, Eds., Glocalized Solutions for Sustainability in Manufacturing. Springer Berlin Heidelberg, 2011.

[14] R. Neugebauer, R. Wertheim, C. Hochmuth, G. Schmidt and M. Dix, vol. 4th CIRP HPC 2010: proceedings of the 4th CIRP International Conference on High Performance Cutting, College International pour L'Etude Scientifique des Techniques de Production. Fraunhofer IWU, oct 2010 .

[15] Y. Wang, B. Hu, T. Cheng, X. Liu, and W. Hu, "Peak-shaving and valleyfilling strategies with UHV introduction based on an improved peaking model," in 2016 International Conference on Cogeneration, Small Power Plants and District Energy (ICUE). IEEE, sep 2016.

[16] H. Ashraf, A. Hassan, U. Khurshid, A. Mahmood, N. Shaheen, Z. A. Khan, U. Qasim, and N. Javaid, "Peak load shaving model based on individual's habit," in 2015 Ninth International Conference on Complex, Intelligent, and Software Intensive Systems, July 2015, pp. 276-282.

[17] G. Parise, L. Parise, A. Malerba, F. M. Pepe, A. Honorati, and P. Chavdarian, "Comprehensive peak-shaving solutions for port cranes," in 2016 IEEE Industry Applications Society Annual Meeting, Oct 2016, pp. 1-7.

[18] P. Horowitz, The art of electronics. New York, NY: Cambridge University Press, 2015.

[19] P. García, "Efecto de las condiciones iniciales sobre la controlabilidad de estado en procesos por lotes," Tesis de Maestría, Universidad Nacional de Colombia, Medellín, Colombia, 2012.

[20] R. Hooke and T. A. Jeeves, "Direct search solution of numerical and statistical problems," Journal of the ACM, vol. 8, no. 2, pp. 212-229, apr 1961.

[21] L. Ljung, System identification : theory for the user. Englewood Cliffs, NJ: Prentice-Hall, 1987.

[22] MATLAB and System Identifiaction Tool Release 2015a, The Mathworks, Inc., Natick, Massachusetts, 2015. 